\title{
HRJ
}

V.3 n.14 (2022)

Recebido: 04/11/2021

Aceito: 01/12/2021

\section{Suspensões Cirúrgicas em um Hospital Público de Médio Porte do Distrito Federal}

\author{
Gilson dos Santos Nascimento ${ }^{1}$ \\ Verônica Santos de Melo ${ }^{2}$ \\ EbertMendonça ${ }^{3}$ \\ FernandaPereiradeOliveira ${ }^{4}$ \\ Heglisson Gustavo da Silva Queiroz ${ }^{5}$ \\ Helen Meirelles Barbosa ${ }^{6}$ \\ Iracema Virginia Noleto ${ }^{7}$ \\ Jacqueline Ramos de Andrade Antunes Gomes ${ }^{8}$ \\ Kerginaldo Severiano de Melo Junior ${ }^{9}$ \\ Laura Fernanda Pereira Silva ${ }^{10}$
}

\begin{abstract}
${ }^{1}$ Enfermeiro Residente do Programa de Residência de Enfermagem em Centro Cirúrgico ESCS/FEPECS/SES/DF

${ }^{2}$ Enfermeira Residente do Programa de Residência de Enfermagem em Centro Cirúrgico ESCS/FEPECS/SES/DF

${ }^{3}$ Enfermeiro Preceptor do Programa de Residência de Enfermagem em Centro Cirúrgico ESCS/FEPECS/SES/DF ${ }^{4}$ EnfermeiraPreceptora do Programa de Residência de Enfermagem em Centro Cirúrgico ESCS/FEPECS/SES/DF

${ }^{5}$ Enfermeiro Preceptor do Programa de Residência de Enfermagem em Centro Cirúrgico ESCS/FEPECS/SES/DF

${ }^{6}$ EnfermeiraPreceptora do Programa de Residência de Enfermagem em Centro Cirúrgico ESCS/FEPECS/SES/DF

${ }^{7}$ EnfermeiraPreceptora do Programa de Residência de Enfermagem em Centro Cirúrgico ESCS/FEPECS/SES/DF

${ }^{8}$ Enfermeira Coordenadora do Programa de Residência de Enfermagem em Centro Cirúrgico ESCS/FEPECS/SES/DF

${ }^{9}$ Enfermeiro Preceptor do Programa de Residência de Enfermagem em Centro Cirúrgico ESCS/FEPECS/SES/DF

${ }^{10}$ EnfermeiraPreceptora do Programa de Residência de Enfermagem em Centro Cirúrgico

ESCS/FEPECS/SES/DF
\end{abstract}

\section{RESUMO}

A administração e o gerenciamento de um centro cirúrgico fazem parte das atribuições dos enfermeiros dessa unidade, cabendo a esses profissionais a tarefa de prover equipamentos e materiais com a finalidade de melhorar as condições de trabalho durante o processo cirúrgico. A suspensão de cirurgia é um importante indicador na gestão de um centro cirúrgico, que deve ser alvo de atenção. A presente pesquisa visa responder a seguinte questão: Quais as principais causas de suspensão de cirurgia e qual a incidência desses cancelamentos? Compreender este acontecimento é fundamental para minimizar gastos públicos e desgastes nos pacientes e familiares. Trata-se de uma pesquisa retrospectiva de natureza descritiva com abordagem quantitativa com o objetivo principal de investigar a incidência e as principais causas de suspensões cirúrgicas em um Centro Cirúrgico (CC) de um hospital público de médio porte localizado no Distrito Federal. O período de coleta de dados será nos meses de 
novembro e dezembro de 2020 e serão levantados dados de cirurgias eletivas realizadas de janeiro a dezembro de 2020.

Palavras-Chaves: procedimentos cirúrgicos operatórios; enfermagem perioperatória indicadores de qualidade em assistência à saúde; centro cirúrgico.

\title{
Surgical suspensions in a medium-sized public hospital in the Federal District
}

\begin{abstract}
The administration and management of a surgical center are part of the duties of nurses in this unit, and these professionals are responsible for providing equipment and materials in order to improve working conditions during the surgical process. Suspension of surgery is an important indicator in the management of a surgical center, which should be the target of attention. This research aims to answer the following question: What are the main causes of surgery suspension and what is the incidence of these cancellations? Understanding this event is essential to minimize public expenses and strain on patients and families. This is a retrospective descriptive study with a quantitative approach with the main objective of investigating the incidence and main causes of surgical suspensions in a Surgical Center (SC) of a medium-sized public hospital located in the Federal District. The data collection period will be in the months of November and December 2020 and data from elective surgeries performed from January to December 2020 will be collected.
\end{abstract}

Keywords: surgical surgical procedures; perioperative nursing quality indicators in health care; surgery Center.

\section{INTRODUÇÃO}

O cuidado é a função básica da enfermagem, que tem como foco o ser humano e suas necessidades biopsicossociais, centrado na assistência integral à saúde. Assim como qualquer ciência, a enfermagem necessita estabelecer e fortalecer sua cultura e linguagem própria, a fim de que, os profissionais entendam suas atribuições e possam assistir as necessidades dos pacientes ${ }^{1}$.

A administração e gerenciamento de um centro cirúrgico fazem parte das atribuições dos enfermeiros dessa unidade, cabendo a esses profissionais a tarefa de prover equipamentos e materiais com a finalidade de melhorar as condições de trabalho durante o processo cirúrgico, com intuito de promover aos pacientes melhores condição de saúde. Para isso faz se necessário um planejamento o mesmo englobe não só as equipes cirúrgicas, mas também os 
demais profissionais ou serviços correlacionados. É necessário também que seja previsto pelos enfermeiros, as mais variadas possíveis intercorrências, relacionadas ou não com o paciente $^{2}$.

Conforme Sodré e Fahl (2014) ${ }^{3}$, suspensão de cirurgia eletiva é definida como sendo qualquer procedimento cirúrgico que programado para certa data não é realizado nesse dia. A circunstância que leva a cada suspensão é verificada no dia seguinte, através das anotações realizadas pela equipe cirúrgica. E está entre as principais consequências da má gestão e gasto de tempo e recursos, o que leva a prejuízos não só a essas unidades de saúde, como também aos pacientes e seus familiares despertando-lhes sentimentos negativos, para com a instituição e profissionais envolvidos.

De acordo, Botozini e carvalho $(2017)^{4}$, a suspensão de cirurgia é um importante indicador na gestão de um centro cirúrgico, que deve ser alvo de atenção de uma equipe multidisciplinar capacitada e empenhada na busca de melhores resultados e prestação de serviços de qualidade. Para isso, a avaliação de desempenho do centro cirúrgico sob o ponto de vista da gestão, buscando aperfeiçoar o desenvolvimento de novos processos, necessita ser avaliado com base em critérios pré-determinados que englobem qualidade, uso indicadores e desempenho da equipe envolvida ${ }^{5}$.

\section{OBJETIVOS}

\section{Objetivo geral}

Identificar as principais causas de suspensão de cirurgia

\section{Objetivos específicos}

Estabelecer a incidência de suspensões cirúrgicas

Relacionar as suspensões com as especialidades cirúrgicas 


\section{METODOLOGIA}

\section{Delineamento}

Trata-se de um estudo retrospectivo, de natureza descritiva e abordagem quantitativa, em que, para sua realização, será feito o levantamento dos dados nos registros arquivados na unidade de Centro Cirúrgico.

Este estudo é de natureza descritiva pois, visa a observação, o registro, a análise e ordenação dos dados, sem manipulá-los, isto é, sem que haja interferência do pesquisador, procura descobrir com que frequência as suspensões de cirurgias, sua natureza e causas ${ }^{6}$.

Além disso, possui a abordagem quantitativa, pois, emprega este tipo de pesquisa técnicas estatísticas, com cálculos matemáticos para a coleta e análise dos dados. Possuindo como diferencial a intenção de garantir a precisão do trabalho realizado, conduzindo a um resultando com poucas chances de distorções ${ }^{7}$.

\section{Cenário do estudo}

O estudo foi realizado no Centro Cirúrgico (CC) de um hospital público de médio porte localizado no Distrito Federal, que presta assistência médico-hospitalar de média complexidade, sendo referência no Sistema Único de Saúde (SUS).

O CC do hospital cenário do estudo realiza uma média de 144 cirurgias eletivas ao mês, e dispõe de sete salas de cirurgia e sete leitos para Sala de Recuperação Pós-Anestésica (SRPA). A programação cirúrgica eletiva é desenvolvida no período das 7 às $19 \mathrm{~h}$, de segunda a sexta-feira. Além disso, realiza uma média de 80 cirurgias de urgência mensalmente.

No CC em questão são atendidos pacientes cirúrgicos das seguintes especialidades: Cirurgia Geral, Ginecologia e Masteologia, Ortopedia, Urologia, Proctologia , Vascular, plástica e oncologia.

Para que o estudo seja fosse nesse CC será solicitada a autorização do responsável técnico pelo setor e do diretor do hospital. 


\section{Coleta de dados}

Os dados foram coletados nos meses de outubro a dezembro de 2020, após aprovação do Comitê de Ética em Pesquisa, a partir de fontes secundárias, sendo elas prontuários eletrônicos dos pacientes e registros hospitalares, assim não have a necessidade de contato ou abordagem aos pacientes. Por esse motivo, foi solicita ao Comitê de Ética em Pesquisa a dispensa de utilização do Termo de Consentimento Livre e Esclarecido. Para a coleta foi utilizado um roteiro estruturado formulado pelo próprio pesquisador).

População e amostra

A população a estuda foi composta por cirurgias eletivas agendadas entre janeiro a dezembro de 2020, no hospital cenário do estudo, que correspondeu a 1026 procedimentos cirúrgicos.

Para o cálculo da amostra considerou-se a média mensal de 144 cirurgias agendadas no período de março a abril de 2020, mês da formulação do presente projeto. Multiplicouse essamédia por doze, uma vez que serão analisadas cirurgias agendadas em 12 meses, totalizando 1728 procedimentos.

\section{Critérios de inclusão}

Foram incluídas cirurgias eletivas de todas as especialidades cirúrgicas agendadas no centro cirúrgico cenário do estudo no período de janeiro a dezembro de 2020.

\section{Critérios de exclusão}

Serão excluídos os procedimentos cirúrgicos de urgência e/ou emergência, cirurgias de pacientes que não tenham em seus prontuários os dados a serem analisados ou que os registros hospitalares de suspensão cirúrgica não tenham sido preenchidos.

Análise dos dados

Os dados coletados serão tabulados no programa Excel, do Microsoft Office ${ }$, e a análise ocorrereu mediante frequências absolutas. A fim de facilitar a compreensão os dados 
serão apresentados em gráficos e/ou tabelas.

\section{Aspectos éticos}

Este projeto foi devidamente submetido ao Comitê de Ética em Pesquisa da Fundação de Ensino e Pesquisa em Ciências da Saúde - CEP/FEPECS. Desde já, a pesquisador se compromete publicamente com as normas preconizadas pela Resolução CNS n n $^{\circ}$, de 12 de dezembro de2012.

\section{Riscos}

Risco de quebra de sigilo Divulgação de dados confidenciaisInvasão de privacidade;

Risco a segurança dos prontuários.Para minimizar os possíveis riscos:

Será mantida a confidencialidade e a privacidade, a proteção dos dados, os quais serão analisados de forma sigilosa, visando a garantia da não utilização das informações em prejuízo das pessoas, inclusive em termos de autoestima, de prestígio e/ou econômicofinanceiro.

O acesso aos prontuários será limitado apenas pelo tempo, quantidade e qualidade das informações específicas para a pesquisa.

Os resultados não permitiram a identificação dos participantes na pesquisa.

\section{Benefícios}

Aos pacientes, os possíveis benefícios proporcionados pelo estudo em questão são indiretos, porém, a melhoria/correção nos serviços de saúde pública beneficia a todos, com o estabelecimento da incidência de suspenção de cirurgias e identificação de suas principais causas há a possibilidade de adequação e prevenção a esses fatores, melhorando assim o desempenho da unidade cc e amenizando prejuízos tanto a administração e funcionários envolvidos como aos pacientes. 


\section{Hipótese}

Sendo as taxas de suspensões de cirurgias um importante indicador para se avaliar o desempenho de uma unidade de centro cirúrgico e auxiliar na identificação de possíveis falhas a serem corrigidas, tona-se necessário o constante acompanhamento/avaliação de desempenho dos serviços prestados nessa unidade, portanto qual a taxa de suspensão de cirurgia no centro cirúrgico?

\section{Cronograma}

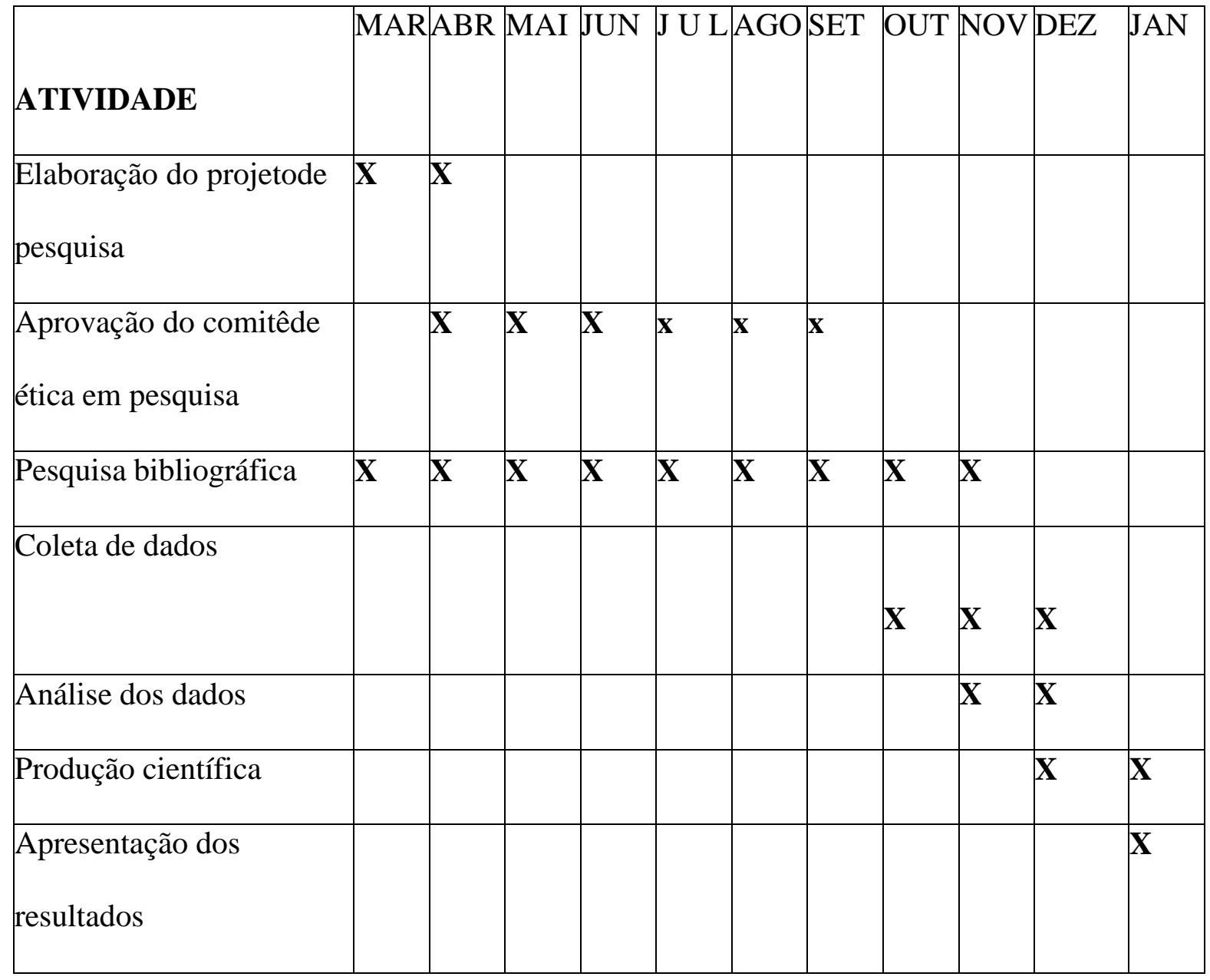

\section{Orçamento}

\begin{tabular}{|l|l|c|}
\hline Descrição & Especificação & Valor (R\$) \\
\hline Material permanente & Computador & 500,00 \\
\cline { 2 - 3 } & Impressora & 2500,00 \\
\hline
\end{tabular}




\begin{tabular}{|l|l|c|}
\hline Material de consumo & Material de escritório & 15,00 \\
\cline { 2 - 3 } & Papelaria & 24,90 \\
\hline Serviços de terceiros & Consultoria de estatística & - \\
\cline { 2 - 3 } & Xerox & - \\
\hline Coleta de dados & Transporte & 270,00 \\
\cline { 2 - 3 } & Alimentação & 120,00 \\
\cline { 2 - 3 } & Telefone/Internet & 100,00 \\
\hline Publicação em periódicos & Revisão de português e outros & - \\
& idiomas & 1279,90 \\
\hline Total & Assinatura do periódico & \\
\hline
\end{tabular}

Obs.: Este orçamento foi custeado em sua íntegra pelo pesquisador.

\section{RESULTADOS / DISCUSSÃO}

No período de janeiro a dezembro de 2020, na unidade em estudo, foram programadas $1.026(100 \%)$ cirurgias eletivas das quais $855(86,3 \%)$ e 171 cirurgias foram canceladas, registrando assim, uma taxa de suspensão de cirurgia nesse período de $16,6 \%$, representado na figura a baixo.

Figura 1 Cirurgias realizadas e canceladas, no período de janeiro a dezembro de 2020.

\section{Cirurgias Eletivas}

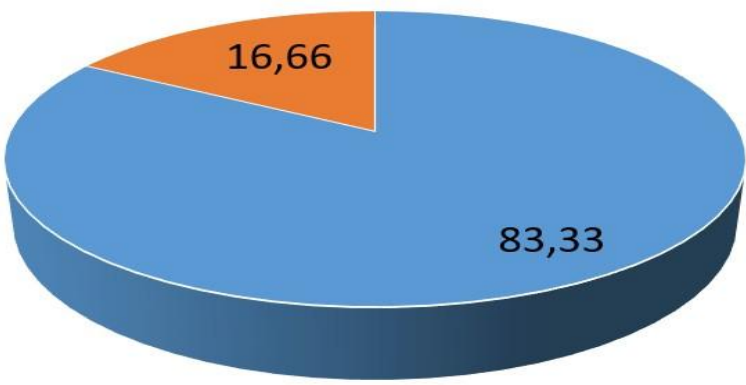


Fonte: banco de dados da pesquisa, 2020.

Os indicadores relacionados à suspensão de cirurgia devem ser monitorados continuamente a fim de subsidiar o planejamento de estratégias de intervenção que visem a redução da taxa de suspensão cirúrgica e assim, minimizando os transtornos causados para os clientes, familiares e instituição ${ }^{8}$.

A taxa de suspensão de cirurgia de $16,6 \%$, identificada na unidade de centro cirúrgico no período em estudo, é considerada moderada, quando comparada com o encontrado na literatura que apresenta uma ampla variação nas taxas de suspensões cirúrgicas, variando ente $0,48 \%$ a $38 \%{ }^{4}$.

Esta taxa identificada no estudo, coincide com as pesquisas realizadas por Macedo et al (2013) $)^{9}$ em um Hospital Público Universitário situado no interior paulista, onde a taxa de suspensão cirúrgica foi de $17,3 \%$, e por Pinheiro et al $(2017)^{10}$ que na UCC de um hospital universitário localizado no interior do estado do Paraná, constatou uma taxa de 18,45\%.

Porém, difere de outra pesquisa realizada, em um hospital público do Distrito federal, por Gomes et tal $(2018)^{11}$, que teve como objetivos calcular a taxa de suspensões de cirurgias canceladas e identificar os fatores determinantes da suspensão de cirurgias eletivas, em um período de dez meses e identificou uma taxa de $33,8 \%$.

Com relação às especialidades, a Tabela 1, a seguir apresenta a frequiência de suspensão segundo a especialidade, analisado, no período de janeiro a dezembro de 2020, na unidade em estudo.

TABELA 1 a seguir apresenta a frequiência de suspensão segundo a especialidade

\begin{tabular}{lllllllllllllll}
\hline Especialidade & Jan & Fev & Mar & Abr & Mai & Jun & Jul & Ago & Set & Out & Nov & Dez & Total & $\%$ \\
\hline Ortopedia & 12 & 9 & 19 & 13 & 13 & 13 & 6 & 0 & 0 & 1 & 0 & - & 86 & 50,2 \\
Geral & 8 & 4 & 4 & 0 & 7 & 2 & 0 & 0 & 0 & 0 & 0 & & 25 & 14,6 \\
Ginecologia & 7 & 8 & 3 & 3 & 3 & 1 & 0 & 0 & 3 & 0 & 1 & - & 29 & 16,9 \\
Urologia & 2 & 2 & 2 & 0 & 0 & 2 & 1 & 0 & 0 & 0 & 0 & - & 9 & 5,2 \\
Proctologia & 0 & 0 & 1 & 1 & 0 & 0 & 1 & 2 & 2 & 0 & 0 & - & 7 & 4,0 \\
Mesologia & 1 & 0 & 6 & 1 & 1 & 0 & 0 & 0 & 0 & 3 & 1 & - & 13 & 7,6 \\
Plástica & 1 & 1 & 0 & 0 & 0 & 0 & 0 & 0 & 0 & 0 & 0 & - & 2 & 1,1 \\
\hline TOTAL & 31 & 24 & 35 & 18 & 24 & 18 & 8 & 2 & 5 & 4 & 2 & - & 171 & 100 \\
\hline
\end{tabular}


Observando a Tabela 1 possível identificar que, dentre as especialidades médicas a que apresentou uma maior frequência de suspensão cirúrgica, no período estudado, foi a ortopedia com uma taxa de 50,2\%, dos procedimentos cancelados, enquanto a ginecologia contribuiu $16,9 \%$ e a geral com $14,6 \%$, dos procedimentos suspensos no período. Com representações menores tivemos amastrologia, a urologia, proctologia e a plástica com as taxas de 7,6\%, $5,2 \%, 4,0 \%$ e $1,1 \%$ respectivamente.

Um estudo realizado em uma Unidade de Centro Cirúrgico do Hospital Universitário do Oeste do Paraná avaliou 3384 cirurgias eletivas e identificou taxa de suspensão de 18,8\%, sendo que as especialidades cirúrgicas que mais apresentaram suspensão de cirurgias foram ortopedia (42,3\%), seguida da geral $(24,5 \%)$, dentre outras especialidades.

As principais justificativas dos motivos geradores de suspensão cirúrgica no Hospital, em estudo, seguem exposto na Tabela 2.

TABELA 2. Distribuição dos principais motivos de suspensão de cirurgias em um hospital público demédio porte do DF

\begin{tabular}{lcc}
\hline \multicolumn{1}{c}{ PRINCIPAIS MOTIVOS } & SUSPENSAS & PERCENTUAL \\
\hline Relacionado a Unidade & $\mathbf{2 5}$ & $\mathbf{1 4 , 6}$ \\
\hline Falta de sala cirúrgica & 2 & 8 \\
Falta de leitos para internação & 6 & 24 \\
Erro na programação cirúrgica & 7 & 28 \\
Falta de exames & 10 & 40 \\
\hline Relacionado a Recursos humanos & $\mathbf{4 4}$ & $\mathbf{2 5 , 7}$ \\
\hline Falta de anestesiologista & 22 & 50 \\
Falta de cirurgião & 10 & 22,7 \\
Avanço de horário & 4 & 9 \\
Mudança de conduta médica & 1 & 2,2 \\
Outros & 7 & 15,9 \\
\hline Relacionado a Materiais & $\mathbf{2 1}$ & $\mathbf{1 2 , 2}$ \\
\hline Falta de material cirúrgico & 18 & 85,7 \\
Falta de material da farmácia & 3 & 14,2 \\
\hline Relacionado a Pacientes & $\mathbf{3 6}$ & $\mathbf{2 1}$ \\
\hline Não comparecimento para internação & 4 & 11,1 \\
Condição clínica desfavorável & 29 & 80 \\
Recusa à realização de cirurgia & 3 & 8,3 \\
\hline Não especificado & $\mathbf{4 5}$ & $\mathbf{2 6 , 3}$ \\
\hline & & $\mathbf{1 0 0}$ \\
\hline TOTAL & $\mathbf{1 7 1}$ & \\
\hline
\end{tabular}

Fonte: banco de dados da pesquisa, 2020 
As principais justificativas dos motivos geradores de suspensão de cirurgias identificadas, na unidade $\mathrm{CC}$, sãos os fatores relacionados aos recursos humanos que abrange uma taxa de $25,7 \%$ das cirurgias canceladas e correspondem a falta de anestesiologista (50\%) e a falta de cirurgião $(22,7 \%)$. Outros fatores importantes a se considerar, são os motivos relacionados aos pacientes correspondente a $21 \%$ das suspensões e englobam as condições clinica desfavorável (80\%), não comparecimento para internação $(11,1 \%)$ e recusa a realização de cirurgia $(8,3 \%)$.

Embora identificado as principais causas de suspensão de cirurgias nessa unidade, faz se importante ressaltar que $26,3 \%$ das cirurgias canceladas não estavam justificadas.

O reconhecimento das principais causas de cancelamento das cirurgias, torna possível um estudo preciso da gestão do indicador de suspensão cirúrgica e como resultado a qualidade de assistência em saúde, mas para isso, é necessário que as informações dispostas nas fontes de pesquisas, prontuários, sejam dispostos de formas completas ${ }^{12}$.

\section{CONCLUSÃO}

Considerando que, as suspensões de cirurgias além de prejuízos as instituições, também afetam aos pacientes e seus familiares, e tendo a enfermagem como base de sua função o cuidado com foco no ser humano e suas necessidades biopsicossociais, é fundamental à atuação do enfermeiro, de centro cirúrgico, como promotor da redução das taxas de suspensões cirúrgicas, promovendo quando necessário, a disponibilidade de materiais, de salas e equipe de enfermagem.

Com o objetivo de propagar a redução das taxas de suspensão de cirurgias faz-se necessário que o enfermeiro se certifique que as informações e recomendações pré-operatórias sejam claras e que o paciente e seus familiares as tenham entendas, assim contribuindo com a redução das causas relacionadas aos pacientes. 
O presente estudo, realizado em um hospital público de médio porte do distrito federal, possibilitou identificar que dentre as 1026 cirurgias eletivas, foram canceladas 171 cirurgias constatando assim, uma taxa de suspensão cirúrgica de 16,6\%, no período. Permitiu também, a identificação das especialidades cirúrgicas, que apresentaram a maior frequência de cancelamentos de seus procedimentos, sendo elas a ortopedia, ginecologia e geral. A principais causas de suspensão de cirurgia nessa unidade, também foram identificadas, sendo os fatores relacionados a recursos humanos principalmente a falta de anestesista o motivo que aparece com maior frequência.

Por fim, ressalta-se a importância do monitoramento contínuo dos indicadores de qualidade no centro cirúrgico, bem como a importância do correto registro das suspensões cirúrgicas nos prontuários e bancos de dados da instituição para que seja possível o gerenciamento, com mais precisão dos motivos, e a promoção da redução da taxa de suspensão cirúrgica.

\section{REFERÊNCIAS}

1. Vale EG, Pagliuca LMF. Construção de um conceito de cuidado de enfermagem: contribuição para o ensino de graduação. Rev Bras Enferm, Brasília. jan-fev. [Internet] 2011 [Citado em 2021 Jul 22]; 64(1): 106-13. Disponível em:

http://www.scielo.br/pdf/reben/v64n1/v64n1a16.pdf.

2. Morgan W, Bernardino E, Wolff LDG. Implicações do cancelamento de cirurgias em centro cirúrgico - estudo descritivo-exploratório. Online Brazilian Journal of Nursing. [Internet] 2010[Citado em 2021 Jul 22]; V. 9 N. 1.Disponível em: http://www.objnursing.uff.br/index.php/nursing/article/view/j.1676-4285.2010.2591/585

3. Sodré RLS, Fahl MAFE. Cancelamento de cirurgias em um hospital público na cidade de SãoPaulo. RAS, Vol. 16, [Internet] 2014 [Citado em 2021 Jul 22].Doi: DOI: 
$10.5327 / Z 1519-1672201400630005$

4. Botazini N, de-Carvalho R. Cancelamento de cirurgias: uma revisão integrativa da literatura. Revista SOBECC [Internet]. 2017 Dez 19; [Citado em 2021 Jul 22]; 22(4): 230244. Disponível em: https://revista.sobecc.org.br/sobecc/article/view/267.

5. Ávila MAG, Gonçalves IR, Martins I, Moyses AM. Cancelamento de cirurgias: uma revisão integrativa da literatura. Revista SOBECC [Internet]. 2012 Jun 30; [Citado em 2021 Jul 22]; 17(2): 39-47. Disponível em: https://revista.sobecc.org.br/sobecc/article/view/163.

6. Prodanov CC, Freitas EC. Metodologia do trabalho científico: métodos e técnicas da pesquisa edo trabalho acadêmico. 2013. $2^{\mathrm{a}}$ ed. P. 52. Novo Hamburgo: Novo Hamburgo Rio Grande do Sul- Brasil

7. Nique W, Laddeira W. Pesquisa de marketing: uma orientação para o mercado brasileiro. SãoPaulo: Atlas, 2014

8. Carvalho T, Sobral C, Marinho P, Ofélia-Llapa-Rodriguez E, Campos M. Suspensão de cirurgias em um hospital universitário. Revista SOBECC [Internet]. 2016 Dez 20; [Citado em 2021 Jul 22]; 21(4): 186-191. Disponível em: https://revista.sobecc.org.br/sobecc/article/view/46

9. Macedo JM, Kano JA, Braga EM, garcia MA; Caldeira SM. Cancelamento de Cirurgias em um Hospital Universitário: Causas e Tempo de Espera para novo Procedimento.Rev. Sobecc, São Paulo[Inernet] 2013. [Citado em 2021 Jul 22]; vol. 18, n. 1. P. 26-34.

Disponível em:< http://www.sobecc.org.br/arquivos/artigos/2012/pdf/ArtigosCientificos/Ano18_n1_jan_mar2013_cancelamento-de-cirurgias-em-um-hospitaluniversitario.pdf

10. Pinheiro SL, Vasconcelos RO, Oliveira JLC, Matos FGOA; Tonini NS, Alves DCI. Taxa de cancelamento cirúrgico: indicador de qualidade em hospital universitário público. Reme -Rev Min Enferm. [Internet] 2017[Citado em 2021 Jul 22]; . Vol. 21. p. 1014. Disponível 
em: http://www.reme.org.br/artigo/detalhes/1150http://www.reme.org.br/artigo/detalhes/1150

11. Gomes J, Franco R, Morais D, Barbosa B. Fatores determinantes para suspensões de cirurgias eletivas em um hospital do Distrito Federal. Revista SOBECC [Internet]. 2018 Dez 20; [Citado em 2021 Jul 22]; 23(4): 184-188. Disponível em: https://revista.sobecc.org.br/sobecc/article/view/419.

12. Rangel ST, Silva JLS, Silva RCLS, Lima A C B, Campos B A, Pereira EBF. Ocorrência e motivosda suspensão de cirurgias eletivas em um hospital de referência. Rev. Enferm. Digit. Cuid. Promoção Saúde . [Internet] 2019 [Citado em 2021 Jul 22]; 4(2):119-123. Dispnível em: https://cdn.publisher.gn1.link/redcps.com.br/pdf/v4n2a07.pdf 